\title{
The Study of Motion Event Model and Cognitive Mechanism of English Fictive Motion Expressions of Access Paths
}

\begin{abstract}
Na Fan
School of Foreign Languages and Cultures, Nanjing Normal University, Nanjing, China

Abstract-This paper studies the cognitive elements in the motion event model of English fictive motion expressions of access paths --- Mover, Motion, Path, Manner, Duration and Distance. It is found that Mover usually refers to some person or some entity with low abstractness; Motion is to illustrate Path; there are path condition and manner condition, which demonstrate the necessity of path and the exclusion of manner; and Duration is essentially to illustrate Distance. Besides, this paper attempts to explore the cognitive mechanism of the fictive motion expressions from the perspectives of conceptual blending, windowing of attention, perspective state and metonymy. It is discovered that the expressions contain the conceptual blending of real motion and the location of the static object, different portions of paths can be windowed, the construal of such expressions is concerned with short distance perspective and sequential scanning mode, and the metonymy mechanism behind them is MOTION FOR PATH.
\end{abstract}

Index Terms - fictive motion expressions of access paths, motion event model, conceptual blending, perspective state, metonymy

\section{INTRODUCTION}

Human being is endowed with subject consciousness, which is embedded in human language, and makes human language subjective to some extent. One of the manifestations of the subjectiveness of human language is the prevalent fictive motion expressions which vividly demonstrate how human being describes the external static world through dynamic perception and description from his/her own point of view.

According to the characteristics of moving paths, Talmy summarizes six types of English fictive motion as follows: emanation paths, pattern paths, advent paths, frame-relative motion, coextension/coverage paths and access paths fictive motions (Talmy, 2000a, p.99-176). This paper will focus on the English fictive motion expressions of access paths.

An access path is a depiction of a stationary object's location in terms of a path that some other entity might follow to the point of encounter with the object. What is factive here is the representation of the object as stationary, without any entity traversing the depicted path. What is fictive is the representation of some entity traversing the depicted path, whether this is plausible or implausible. (Talmy, 2000a, p.136) For example:

(1) a. The bakery is across the street from the bank. (Talmy, 2000a, p.137)

b. There is a beautiful chapel across the river, through the meadow, and (then) over the hill. (Matsumoto, 1996a, p.365)

(1a) and (1b) are both fictive motion expressions of access paths. In (1a), the location of the bakery is concerned with the motion of some person or some entity commencing from the bank, crossing the street, and then terminate at the bakery. In (1b), the location of the chapel is related with the motion of some entity crossing the river, running through the meadow and climbing over the hill.

The phenomenon of fictive motion in language is based on the motion event model which exists everywhere in the real world. This paper will attempt to study the motion event model and the cognitive mechanisms behind English fictive motion expressions of access paths from the perspectives of conceptual blending, perspective state and metonymy.

\section{Motion Event Model}

On the basis of the cognitive study of motion event model conducted by Talmy $(2000 \mathrm{~b})$ and Fillmore $(1982,1985)$, it is concluded that there are five cognitive elements in the motion event model of English fictive motion expressions of access paths, which are Mover, Motion, Path, Manner, Duration and Distance. The following will study the five elements respectively from the cognitive perspective.

A. Mover

In English fictive expressions of access paths, the mover can usually reach the described location. For instance:

(2) a. His garden is across the fence. 
b. */OK His office is \{across / through \} this wall. (Matsumoto, 1996a, p.368)

(2a) is acceptable because the fence can be crossed by someone; while (2b) is unacceptable because the wall is so hard for some common people to go through.

Here are some other examples:

(3) a. * The village is over the valley from us.

b. ? The cloud is 1,000 feet up from the ground.

c. Up above are branches of a big tree. (Takahashi, 2005, p.9)

(3a) is not acceptable because human beings can not fly over the valley like birds. (3b) is also questionable because we can not reach the cloud 1,000 feet up in the sky unless we take airplane. However, (3c) is acceptable because under some circumstance we may reach the branches of a tree overhead.

All in all, in English fictive motion expressions of access paths, mover usually refers to some person or some concrete entity, which demonstrates that mover in the expressions is of low abstractness.

\section{B. Motion}

In English fictive motion expressions of access paths, the fictive motion process is usually manifested by path prepositions, while not the locative prepositions (Matsumoto, 1996a, p.365). For instance:

(4) There is a lake \{halfway / twenty miles\} across the desert. (Matsumoto, 1996a, p.365)

In (4), the lake is located at some point of the moving process or path of crossing the desert. The preposition "across the desert" is used to illustrate the moving process but not simply describe the location.

In English fictive motion expressions of access paths, motion is mainly illustrated by prepositional phrases that emphasize on the moving path. In another word, motion is mainly to illustrate the motion path but not to describe the motion manner.

C. Path

In English fictive motion expressions of access paths, the path is usually manifested by prepositional phrases. For example:

(5) a. The bike is parked across the street.

b. There is a lake through the forest. (Matsumoto, 1996a, p.359)

The prepositional phrases "across the street" and "through the forest" in (5a) and (5b) are used to manifest the motion paths respectively.

It is found that in English fictive motion expressions of access paths there is a path condition, which means some property of the path of motion must be expressed (Matsumoto, 1996b, p.194; Fan, 2011, p.107, 2012). The path is closely related with location, which is the necessary element in the expressions.

Moreover, it is found that the sequence of path elements in the expressions is consistent with that in the cognitive world, namely they are displayed in some order of Source- Path- Goal (S-P-G) in the expressions. For example:

(6) There is a beautiful chapel across the river, through the meadow, and (then) over the hill. (Matsumoto, 1996a, p.365)

The sequence of path elements in (6) is "across the river -- through the meadow -- over the hill", which coincides with the path sequence in the cognitive world.

It is believed that the consistence of the linguistic representations and cognitive order of path sequence embodies the principle of grammatical iconicity. According to the principle, linguistic representations are always in accordance with human cognition. Therefore, based on the cognitive experience of human beings, there is such a relatively fixed cognitive model of motion path as S-P-G pattern, from which language is organized and produced.

\section{Manner}

In English fictive motion expressions of access paths, the manner is usually related with the path to indicate the locations. For example:

(7) a. The hotel is 15 minutes down the road \{by car / on foot / at that speed .

b. The hotel is a 15-minute \{drive /walk / run \} down the road. (Matsumoto, 1996a, p.367)

The manners of "by car / on foot / at that speed" in (7a) and "drive /walk / run" in (7b) indicate a certain moving speed respectively. Combined with the duration "15 minutes", the manners illustrate the moving distance, so that they can help define the exact location of the hotel.

The above shows that in English fictive motion expressions of access paths, besides the path condition there is a manner condition as well, which means no property of the manner of motion can be expressed unless it is used to represent some correlated property of the path (Matsumoto, 1996b, p.194; Fan, 2011, p.108, 2012). The expressions usually emphasize on the motion path but not the motion manner, therefore, the manner are generally used to illustrate the path.

\section{E. Duration and Distance}

There are some descriptions of moving distance in English fictive motion expressions of access paths. For instance:

(8) His house is halfway through the increasingly dense forest. (Matsumoto, 1996a, p.366) 
In (8), "halfway through the increasingly dense forest" indicates the moving process and moving distance.

Besides, even if without any concrete moving manner in the expressions, there may be some descriptions of duration as long as the producer and receiver of the expressions have the same hypothesis of moving manner and speed. For example:

(9) The city lies many hours across the desert. (Matsumoto, 1996a: 366)

The duration of "many hours" in (9) actually demonstrates the moving distance. The question raised from (9) should be concerned with distance (see (10a)), but not duration (see (10b)).

(10) a. How far across the desert does the city lie?

b. *How long across the desert does the city lie? (Matsumoto, 1996a, p.366)

(10a) is acceptable because the question word "how far" is relevant to moving distance; while (10b) is unacceptable because the question word "how long" is only involved with duration.

Moreover, the descriptions of duration can be further specified. For example:

(11) a. There is a village 25 minutes through the forest.

b. The hotel is only three minutes down the road.

c. There is a bridge 15 minutes down the footpath. (Matsumoto, 1996a, p.367)

In (11a) - (11c), "25 minutes", "three minutes" and "15 minutes" are the specified descriptions of duration.

In short, in some cases there are descriptions of moving duration and distance in English fictive motion expressions of access paths, and the duration fundamentally plays the role of specifying moving distance.

\section{Cognitive Mechanisms}

There are specific cognitive mechanisms behind English fictive motion expressions of access paths. The following will study the cognitive mechanisms from the perspectives of conceptual blending, perspective state and metonymy.

\section{A. Conceptual Blending}

The construal of fictive motion expressions is involved with four mental spaces --- Input Space 1 (Input 1), Input Space 2 (Input 2), Generic Space and Blended Space. First, the correspondent elements of Input 1 and Input 2 have some mapping relationship, so that they form a kind of cross mapping between different spaces. Second, the shared elements of Input 1 and Input 2 are projected into the generic space which reflects the common abstract structure of Input 1 and Input 2. Last, some of the elements in Input 1 and Input 2 are projected into blended space, and form a new emergent structure.

Conceptual blending theory can be used to explore the cognitive mechanism of English fictive motion expressions of access paths. Sentence (12) is taken as an example to analyze the conceptual blending process of such kind of fictive motion expressions.

(12) The bike is across the street. (Matsumoto, 1996a, p.364)

The construal of (12) is involved with four mental spaces: (1) Input 1, which embodies the real motion of crossing the street; (2) Input 2, which includes the location of the static bike; (3) Generic space, which includes the correspondent elements in Input 1 and Input 2. For example, the location of "across the street" in Input 1 and the location of "bike" in Input 2 have something in common with respect to space, so they are projected into the generic space. (4) Blended space, which contains some elements in Input 1 and 2. For instance, the motion of "crossing the street" in Input 1 and the location of "bike" in Input 2 are both projected into the blended space, thus producing the emergent structure that includes both the motion of some mover and the location of the bike.

Fig. 1 demonstrates the conceptual blending process of English fictive motion expressions of access paths.

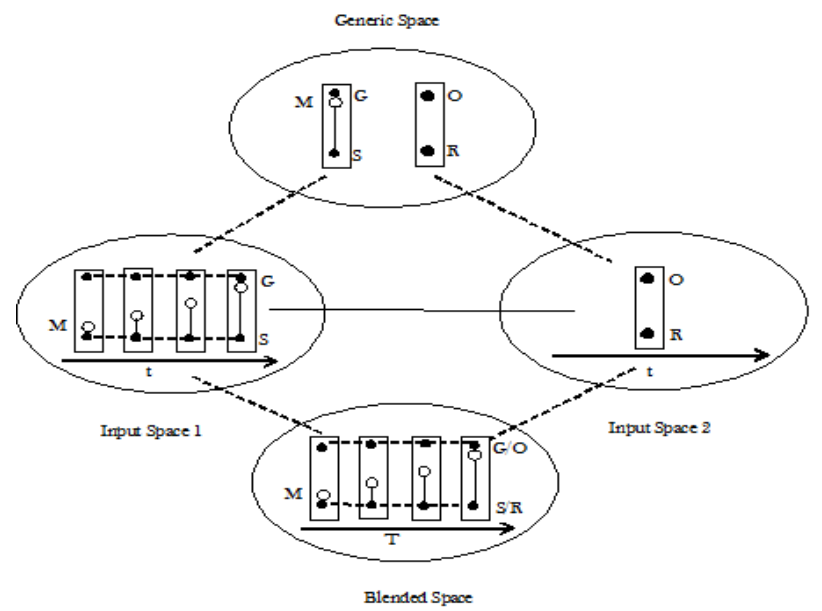

Figure 1 Conceptual Blending of Fictive Motion Expressions of Access Paths ( $\mathrm{S}=$ Source; $\mathrm{G}=\mathrm{Goal} ; \mathrm{M}=$ Mover; $\mathrm{O}=$ Object; $\mathrm{R}=$ Reference) 
In Fig. 1, the two solid circles in Input 1 represent Source and Goal respectively, and the hollow circle represents Mover. The two solid circles in Input 2 represent Object and Reference separately. As is shown, Mover moves from Source to Goal on the time axis in Input 1. While in Input 2, the Object is located in a certain location at some point on the time axis relative to Reference. The correspondent elements in Input 1 and Input 2, such as the Goal of Mover in Input 1 and the location of Object in Input 2 share something in common in regard to space, so they are projected into the Generic Space. Based on the Generic Space, some of the elements in Input 1 and Input 2, such as the motion in Input 1 and the location in Input 2 are both projected into the Blended Space, thus forming an emergent structure. In the Blended Space, the motion of Mover from Source to Goal is used to represent the location of Object relative to Reference.

\section{B. Windowing of Attention}

Linguistic form can direct the distribution of one's attention over a referent scene in a certain type of pattern, the placement of one or more windows of greatest attention over the scene, in a process that can be termed the windowing of attention. (Talmy, 2000a, p.258) A referent scene that is sequential in nature or that has been sequentialized conceptually can have a window of strongest attention placed over its beginning, middle, or end portion --- or may have initial, medial, or final windowing. (Talmy, 2000a, p.259)

The construal of motion event is concerned with various types of windowing, such as path-windowing, causal-chain windowing, phase-windowing, participant-interaction windowing and multiple nested windowing. The construal of English fictive motion expressions of access paths is involved with path-windowing.

According to Talmy (2000a, p.265), the path can be divided into three types: open paths, closed paths and fictive paths. An open path refers to a path that is described by an object physically in motion in the course of a period of time, that is conceptualized as an entire unity thus having a beginning and an end, and whose beginning point and ending point are at different locations in space.

The following example is concerned with the open-path type with various patterns of windowing and gapping imposed on it.

(13) The crate that was in the aircraft's cargo bay fell ---

a. With maximal windowing over the whole of the so-conceived entire path

--- out of the plane through the air into the ocean.

b. With gapping over one portion of the path

i. Medial gapping = initial + final windowing

--- out of the plane into the ocean.

ii. Initial gapping $=$ medial + final windowing

--- through the air into the ocean.

iii. Final gapping = initial +medial windowing --- out of the airplane through the air.

c. With windowing over one portion of the path

i. Initial windowing $=$ medial + final gapping

--- out of the airplane.

ii. Medial windowing = initial +final gapping

--- through the air.

iii. Final windowing $=$ initial + medial gapping

--- into the ocean.

(Talmy, 2000a, p.266)

(13a) presents the event with maximal windowing over the whole of the conceptually complete path, while (13b) presents three forms of gapping over one portion of the path and (1c) presents three forms of windowing over one portion of the path.

The fictive path refers to the "trajectory" exhibited by a person's focus of attention shifting over a conceived scene. Talmy analyzed an English construction of "X BE across $Y$ " that directs one's focus of attention along a spatial path in this way. This construction belongs to a type of fictive motion expressions of access paths, which specifies a fictive equivalent of an open path in which one's focus of attention starts from point Z, traverses the extent of Y, and that lastly terminates at point $X$.

The following example demonstrates the maximal windowing over the entire path and the windowing over some portions of the path.

(14) a. With maximal windowing

i. My bike is across the street from the bakery.

ii. Jane sat across the table from John.

b. With medial gapping = initial + final windowing

i. My bike is across from the bakery.

ii. Jane sat across from John.

c. With initial gapping $=$ medial + final windowing 


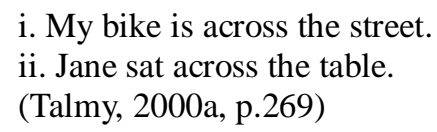

(14ai) and (14aii) describe the entire path of the focus of attention, which starts from bakery, traverses the street and terminates at the location of "my bike". (14b) and (14c) indicate some portions of the path of the focus of attention. In (14bi) and (14bii), the initial and final portions of the path are windowed while the medial portion is absent; In (14ci) and (14cii), the medial and final portions of path are windowed while the initial portion is absent.

Talmy compared the two English constructions "X BE across Y from Z" and "X BE between Y and Z". These two constructions specify a complex spatial schema that includes two reference point (the Ground objects $Y$ and $Z$ ). The "between" construction calls for a stationary distal perspective point with global scope of attention over the spatial schema as a whole, while the "across from" construction specifies a moving proximal perspective point with local scope of attention on elements of the schema taken in sequence. (Talmy, 2000a, p.269)

All in all, in the construal of English fictive motion expressions of access paths, the initial or the medial portions of path may be windowed, while the final portion of path must be windowed, because the final portion of path is closely related with the location of the described objects and thus plays an important role in the conceptualization of such expressions.

\section{Perspective State}

Perspective state, which is also called "perspective mobility" by Talmy (2000a, p.70), mainly refers to the moving or stationary state of perspective. Generally speaking, the stationary state of Object is involved with the long distance perspective, while the moving state with the short distance perspective. The former is usually concerned with the global scope of attention and the latter is related to the local scope of attention. Perspective state is divided into synoptic mode and sequential mode by Talmy (2000a, p.70). The synoptic mode is connected with the stationary long distance perspective and global scope of attention, while sequential mode is in connection of the moving short distance perspective and local scope of attention.

The similar notion of "perspective state" is "scanning mode" put forward by Langacker (1987). Langacker summarizes the scanning mode into two categories: summary scanning and sequential scanning. The former refers to that every part of the conceptualized content are activated, processed simultaneously and thus forming a whole gestalt. The latter means that the objects or events are processed in sequence by human cognition. The summary scanning emphasizes the global state of event, while the sequential scanning accentuates the connective relationship or process of event.

What is adopted in the construal of English fictive motion expressions of access paths is the short distance perspective that is related with moving and the sequential scanning mode of local scope of attention. The motion and paths (such as Source, Path, Direction and Goal) can be sequentially scanned, thus constructing a whole moving path, and the goal of which is related with the location of the object. For example:

(15) The chapel is across the river from us.

In (15), the whole moving path is constructed through the sequential scanning of Source (form us) and Path (across the river), thus explicating the location of the chapel.

\section{Metonymy}

Metonymy is not only an important figure of speech but also the reflection of human cognitive mechanism. Based on the generation mechanism of metonymy, Radden \& Kövecses (1999, p.30) put forward a system of "generation relationship of metonymy". It is believed that the generation of metonymy is decided by two conceptual structures: (1) the relation between the whole ICM and each element in it; (2) the relation between each element in the whole ICM.

The first conceptual structure, concerned with people or event, refers to the substitution of the whole for parts or parts for the whole, which generates a kind of referential metonymy. The second conceptual structure, concerned with event or state, refers to the substitution of part for part, which generates a kind of predication metonymy (Panther \& Thornburg, p.1999). Take the ACTION cognitive model as an example, this model consists of such elements as Agent, Object, Action, Tool, Manner and Result, which are connected with each other. Sometimes for example, Agent, Tool, Result and Manner can be used to refer to Action respectively, and Action can be used to refer to Result.

Therefore, it is regarded that what is involved in the fictive motion expressions is the predication metonymy that embodies the substitution relationship of part for part. For instance, the cognitive model of motion event consists of such elements as Mover, Motion, Path and Manner, which embrace a certain kind of substitution between each other, for example "Motion for Path". This kind of relationship promotes the generation of metonymy in fictive motion expressions.

The motion event in fictive motion expressions of access paths is composed of such elements as Mover, Motion, Path and Manner. The relationship of motion and path arouses the metonymy of "Motion for Path". For example:

(16) There is a gas station toward the end of the street. (Matsumto, 1996b, p.364)

In (16), the connection of motion and path triggers the metonymy of " the motion of going toward the end of the street' for 'the path of going toward the end of the street"'.

Fig. 2 demonstrates the metonymy mechanism of fictive motion expressions of access paths. 


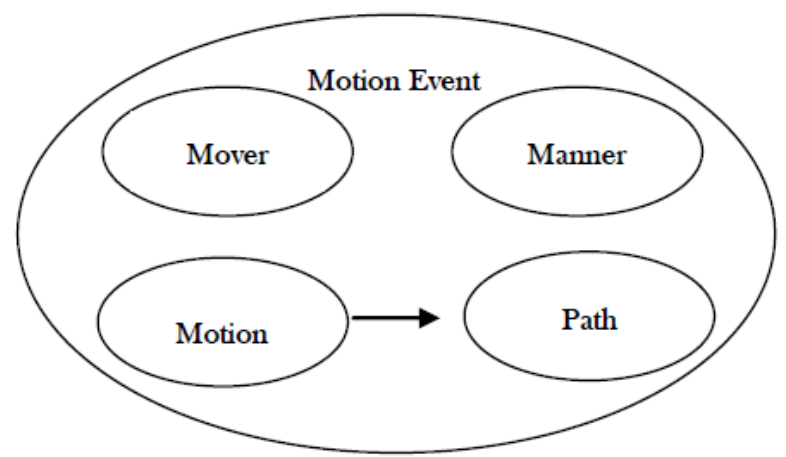

Figure 2 Metonymy Mechanism of Fictive Motion Expressions of Access Paths: MOTION FOR PATH

In Fig. 2, the big circle represents the cognitive model of motion event, and the small circles represent various elements in the cognitive model. Motion, as one of the elements in the cognitive model activates another element --Path, thus generating the metonymy of MOTION FOR PATH.

In fact, the metonymy of MOTION FOR PATH belongs to another superior metonymy, namely ACTION FOR RESULT, in which the motion is used to represent the result.

\section{CONCLUSION}

The study of fictive motion expressions is of great meaning to discover the cognitive mechanisms behind linguistic representations. Based on the cognitive study of elements in the motion event model of English fictive motion expressions of access paths, it is found that Mover is of low abstractness because it only refers to some concrete person or entity. Due to the path condition and manner condition, which demonstrate the necessity of path and the exclusion of manner, Motion and Manner can only be used to describe Path. Meanwhile, Duration is essentially to illustrate Distance, which is also concerned with Path to some extent.

Through the study of the cognitive mechanism of English fictive motion expressions of access paths, it is discovered that the construal of such expressions is concerned with the conceptual blending of real motion and the location of the stationary object, the windowing of attention of different portions of paths, the short distance perspective and sequential scanning mode, and the metonymy of MOTION FOR PATH.

The study of motion event model and cognitive mechanism of English fictive motion expressions of access paths will surely be beneficial in the exploration of the relationship between language and cognition.

\section{ACKNOWLEDGMENT}

This work is supported in part by College Philosophy and Social Sciences Foundation of Jiangsu Education Department, project number: 2013SJB750028 and A Project Funded by the Priority Academic Program Development of Jiangsu Higher Education Institutions (PAPD).

\section{REFERENCES}

[1] Fan, N. (2011). Path and manner of English fictive motion expressions of coextension paths. Journal of Dalian Maritime University (Social Science Edition), 2, 106-109.

[2] Fan, N. (2012). A Cognitive study of Chinese fictive motion expressions in the motion event model. Ph.D. dissertation, Beijing Foreign Studies University.

[3] Fillmore, C. J. (1982). Frame semantics. In The Linguistic Society of Korea (Ed.), Linguistics in the morning calm. Seoul: Hanshin, 111-137.

[4] Fillmore, C. J. (1985). Frames and the semantics of understanding. Quaderni di Semantica, 2, 222-254.

[5] Langacker, R. W. (1987). Foundations of cognitive grammar (vol. 1). Stanford, CA: Stanford University Press.

[6] Matsumoto, Y. (1996a). How abstract is subjective motion? A comparison of coverage path expressions and access path expressions. In A. Goldberg (Ed.), Conceptual structure, discourse, and language. Stanford. CA: CSLI Publications, 359-373.

[7] Matsumoto, Y. (1996b). Subjective motion and English and Japanese verbs. Cognitive Linguistics, 2, 183-226.

[8] Panther, K.-U. \& L. Thornburg. (1999). The potentiality for actuality metonymy in English and Hungarian. In K. Panther \& G.. Radden (Eds.). Metonymy in language and thought. Amsterdam, Netherlands/Philadelphia, PA: John Benjamins Publishing Company, 333-357.

[9] Radden, G. \& Z. Kövecses. (1999). Toward a theory of metonymy. In K. Panther \& G. Radden (Eds.). Metonymy in language and thought. Amsterdam, Netherlands/Philadelphia, PA: John Benjamins Publishing Company, 17-59.

[10] Takahashi, K. (2005). Construal of fictive motion categories: a contrastive study between English and Thai. In The 9th International Cognitive Linguistics Conference, Seoul, July 17-22.

[11] Talmy, L. (2000a). Toward a cognitive semantics vol. 1: Concept structuring systems. Cambridge, MA: MIT Press. 
[12] Talmy, L. (2000b). Toward a cognitive semantics vol. 2: Typology and process in concept structuring. Cambridge, MA: MIT Press.

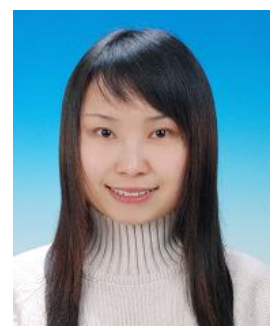

Na Fan, Xuzhou, 1978, Ph.D., English language and literature, Beijing Foreign Studies University, Beijing, China, 2012.

She is now a lecturer at the School of Foreign Languages and Cultures, Nanjing Normal University, Nanjing, China. Her main publications are as follows: A cognitive study of Chinese fictive motion expressions in the motion event model (Nanjing, Jiangsu: Nanjing University Press, 2014) (in press); The comparative study of Chinese and English fictive motion expressions of access paths. Journal of Xi'an International Studies University (CSSCI), 2014, 01; Abstractness in English coextension paths fictive motion expressions. Journal of Foreign Language Education (CSSCI), 2012, 02. Her current research interests are cognitive linguistics and applied linguistics. 